\title{
Crystal-Structure Contribution to the Solid Solubility in Transition Metal Alloys
}

\author{
Ruban, Andrei; Skriver, Hans Lomholt; Nørskov, Jens Kehlet
}

Published in:

Physical Review Letters

Link to article, DOI:

10.1103/PhysRevLett.80.1240

Publication date:

1998

Document Version

Publisher's PDF, also known as Version of record

Link back to DTU Orbit

Citation (APA):

Ruban, A., Skriver, H. L., \& Nørskov, J. K. (1998). Crystal-Structure Contribution to the Solid Solubility in Transition Metal Alloys. Physical Review Letters, 80(6), 1240-1243.

https://doi.org/10.1103/PhysRevLett.80.1240

\section{General rights}

Copyright and moral rights for the publications made accessible in the public portal are retained by the authors and/or other copyright owners and it is a condition of accessing publications that users recognise and abide by the legal requirements associated with these rights.

- Users may download and print one copy of any publication from the public portal for the purpose of private study or research.

- You may not further distribute the material or use it for any profit-making activity or commercial gain

- You may freely distribute the URL identifying the publication in the public portal

If you believe that this document breaches copyright please contact us providing details, and we will remove access to the work immediately and investigate your claim 


\title{
Crystal-Structure Contribution to the Solid Solubility in Transition Metal Alloys
}

\author{
A. V. Ruban, H. L. Skriver, and J. K. Nørskov \\ Center for Atomic-scale Materials Physics and Department of Physics, Technical University of Denmark, \\ DK-2800 Lyngby, Denmark
}

(Received 8 August 1997)

\begin{abstract}
The solution energies of $4 d$ metals in other $4 d$ metals as well as the bcc-hcp structural energy differences in random $4 d$ alloys are calculated by density functional theory. It is shown that the crystal structure of the host plays a crucial role in the solid solubility. A local virtual bond approximation accounts for the calculated solution energies and explains the substantial reduction in structural energy caused by randomness. [S0031-9007(97)05224-1]
\end{abstract}

PACS numbers: 61.66.Dk, 73.30.+y

The "macroscopic atom" model of enthalpy effects in alloys developed by Miedema and co-workers [1,2] has been highly influential in practical metallurgy work. One important aspect of the model is the possibility to predict impurity solution energies in solid solutions about which very little is known even in binary systems except for a few experimental values [2,3]. Even today, with the advent of accurate first-principles computer techniques calculations of impurity solution energies have only been performed for a few systems [4-7], and the general trends across the periodic table have not been established.

Miedema and Niessen [2,8,9] discuss in their pioneering work three contributions to the impurity solution energy: a chemical contribution which includes "liquidlike" interactions, a relaxation contribution arising from atomic size mismatch of the host atoms and impurity, and a structural contribution, i.e., an additional contribution due to the fact that the crystal structure of the host is fixed during solution. It is the structural contribution which is the subject of the present paper, and we note that intuitively it does not seem to be pronounced, let alone dominant. However, from the analysis of $\mathrm{Zr}$-based phase diagrams Miedema and Niessen [8] find that the solubilities of other transition metals in the two structural forms of $\mathrm{Zr}$, hcp, and bcc, differ dramatically and thereby provide the first indication that the structural contribution to the heat of solution may, in fact, play an important role in determining the phase diagrams of transition metal alloys.

The model of Miedema and Niessen for the structural contribution to the solution energy in transition metal alloys exploit the fact that the structural energy differences in the pure transition metals may be considered canonical [10] functions of the valence $d$-band occupation number $N_{d}$ [11-13]. Hence, in the spirit of the virtual crystal approximation (VCA) they assume that for a given crystal structure the $d$ bands in a random $A_{1-c} B_{c}$ alloy are those of an "average" pure metal and use for the structural energy differences in the alloy the canonical curves corresponding to the pure metal bands but occupied by the concentration weighted average $d$ occupation $(1-c) N_{d}^{A}+c N_{d}^{B}$. At first sight, this appears reasonable, at least in the dilute limit of the impurity concentration $c$, and indeed the model turns out to be quite successful in explaining the crystal-structure effect on the solid solubility in the Zr-Pd system [8].

In the present Letter, we demonstrate on the basis of extensive density functional theory (DFT) calculations [14] of solution energies in $4 d$ transition metal alloys that the crystal-structure contribution plays a major role in the solid solubility of transition metals in transition metals. We also develop a model, the virtual bond approximation (VBA), based on a local description of the bonding in dilute alloys, which is able to account qualitatively and semiquantitatively for the solution energy differences between different host crystal structures as well as for the structural energy differences of random alloys. This subject is not treated within ordinary theory of phase transformations in alloys [15] nor is it considered in first-principles calculations of phase diagrams $[16,17]$.

We present in Fig. 1 a database of solution energies of the $4 d$ metals in other $4 d$ metals calculated using the definition

$$
E_{\mathrm{sol}}^{\alpha}(B \rightarrow A)=\left.\frac{\partial E_{A_{1-c} B_{c}}^{\alpha}}{\partial c}\right|_{c=0}+E_{A}^{\alpha}-E_{B}^{\beta},
$$

of the energy in the dilute limit of metal $B$ in an $A$ host having an $\alpha$ structure [6]. Here, $E_{A_{1-c} B_{c}}^{\alpha}, E_{A}^{\beta}$, and $E_{B}^{\beta}$ are the total energies of the $A_{1-c} B_{c}$ alloy and the pure metals in the $\alpha$ and $\beta$ structures, respectively. The details of the calculations are given in Refs. $[18,19]$. We do not include the effect of lattice relaxations around the impurity but for the present systems with moderate size mismatches they are small [20], and we expect the results to provide at least a qualitative description of the "chemical" and crystalstructure contributions.

At first sight the results in Fig. 1 do not seem to exhibit any recognizable trends. If, for instance, we employ the qualitative theories of bonding in transition metal alloys, based on tight-binding or Friedel-like considerations [2123], we expect the solution energies to be smooth functions of the average number of $d$ electrons of the alloy components or their difference, $\Delta N_{d}=N_{d}^{A}-N_{d}^{B}$. This is obviously not the case and the reason for the irregular 


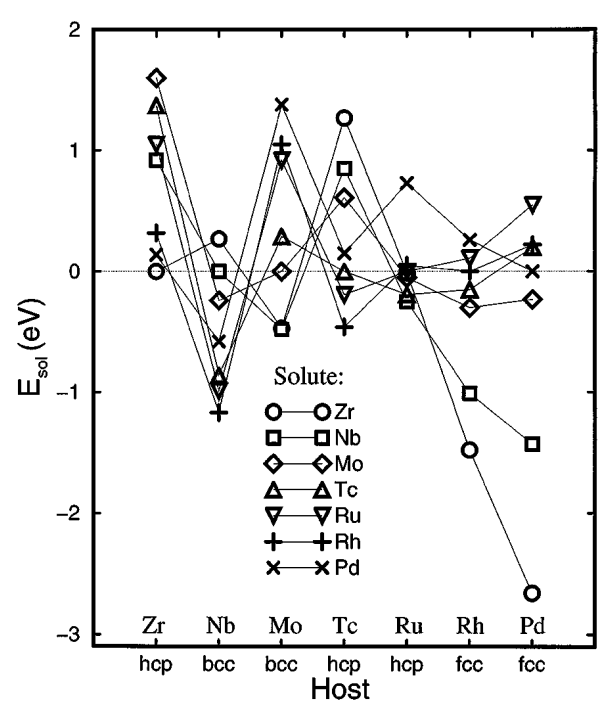

FIG. 1. Solution energies for $4 d$ metals in $4 d$ metal hosts calculated by (1). The thin lines connect results for a particular impurity and the host crystal structure is indicated at the abscissa.

changes of the solution energies from one host to the next cannot be explained on the basis of considerations which do not include structural effects.

Closer inspection of Fig. 1 reveals a very simple rule which makes the results systematic and easy to describe. Consider the elements ordered according to $d$ occupation. Then, if the element next to the host in the direction of an impurity has the same crystal structure as the host the solution energy increases, otherwise it decreases. As an example let us consider impurity solution energies in bcc Mo. The neighboring element on the right-hand side of Mo is hcp Tc, and we observe that all the elements from $\mathrm{Tc}$ to Pd have positive solution energies in Mo. Conversely, the neighboring element on the left-hand side is bcc $\mathrm{Nb}$ and both $\mathrm{Nb}$ and $\mathrm{Zr}$ have negative solution energies. In fact, the rule works so well, especially at the beginning of the series, that one may safely conclude that the solution energies of transition metals in $\mathrm{Zr}, \mathrm{Nb}, \mathrm{Mo}$, and $\mathrm{Tc}$ are almost solely determined by the crystal-structure contribution.

To show that structure plays an important role for the solid solubility of transition metals we discuss the firstprinciples local density approximation (LDA) results on the basis of the difference in solution energy of an impurity in a given host of different crystal structures defined by

$$
\Delta E_{\mathrm{sol}}^{\alpha-\beta} \equiv E_{\mathrm{sol}}^{\alpha}-E_{\mathrm{sol}}^{\beta}=\left.\frac{\partial \Delta E_{A_{1-c} B_{c}}^{\alpha-\beta}}{\partial c}\right|_{c=0}+\Delta E_{A}^{\alpha-\beta},
$$

where $\Delta E_{A_{1-c} B_{c}}^{\alpha-\beta}$ and $\Delta E_{A}^{\alpha-\beta}$ are the structural energy difference in the random $A_{1-c} B_{c}$ alloy and the host, respectively. We further write the total energy of the alloy in terms of two-body potentials (a generalization to manysite interactions is straightforward),

$$
\begin{aligned}
E_{A_{1-c} B_{c}}^{\alpha}= & v^{(0)}+(1-c)^{2} v_{A A}^{(2-\alpha)} \\
& +2 c(1-c) v_{A B}^{(2-\alpha)}+c^{2} v_{B B}^{(2-\alpha)} .
\end{aligned}
$$

Here, $v^{(0)}$ is the on-site term and $v_{X Y}^{(2-\alpha)}$ is obtained as the sum over the whole lattice of pair potentials acting between $X$ and $Y$ atoms. In particular, $v_{A B}^{(2-\alpha)}=\frac{1}{2} \sum_{i \neq j} V_{A B}^{(2)}\left(R_{i}-\right.$ $R_{j}$ ), where $V_{A B}^{(2)}$ are structure-independent potentials defined, for instance, by Moriarty [24] and the sums run over the lattice sites in the $\alpha$ structure.

From (2) and (3) we obtain

$$
\Delta E_{\mathrm{sol}}^{\alpha-\beta}=2\left[v_{A B}^{(2-\alpha)}-v_{A B}^{(2-\beta)}\right]-\left[v_{A A}^{(2-\alpha)}-v_{A A}^{(2-\beta)}\right],
$$

where the last term in the square brackets now represents the $\alpha$ - $\beta$ structural-energy difference in the host $A$. To continue, we model the interaction potentials between different kinds of atoms represented in the first term of (4) by the assumption that they are canonical functions of the average number of $d$ electrons in an $A B$ bond. This we call the virtual bond approximation, and it means that an $n$-body potential of $k A$ atoms and $n-k B$ atoms, $v_{A A \cdots B B}^{(n-\alpha)}$, is equal to the $n$-body potential of a pure metal $C, v_{C \ldots C C}^{(n-\alpha)}$ with the number of $d$ electrons given by $N_{d}^{C}=$ $\frac{1}{n}\left[k N_{d}^{A}+(n-k) N_{d}^{B}\right]$. The so-defined VBA is equivalent to the VCA used, e.g., by Miedema and co-workers [2], when the dominant interactions in the alloy are $n$-body potentials which involve bonding in the entire crystal, i.e., $n \rightarrow \infty$. A similar model [25] has been used with success to explain the site substitutional behavior of all $d$ impurities in $\mathrm{Ni}_{3} \mathrm{Al}$ [26].

In the VBA pair-potential $\left(v^{(2)}\right)$ approximation we now have

$$
\Delta E_{\mathrm{sol}}^{\alpha-\beta}=2 \Delta E_{C}^{\alpha-\beta}\left(N_{d}^{C}\right)-\Delta E_{C}^{\alpha-\beta}\left(N_{d}^{A}\right),
$$

where $N_{d}^{C}=\frac{1}{2}\left(N_{d}^{A}+N_{d}^{B}\right)$ and $\Delta E_{C}^{\alpha-\beta}$ is the canonical structural energy difference curve included in Fig. 2. As we shall demonstrate below this simple expression contains the physics of the structural contribution to the solid solubility.

The structural difference in the solution energies of the $4 d$ metals obtained directly from first principles are plotted in Fig. 2 as functions of $N_{d}^{C}$. We immediately observe that the values in the figure are unexpectedly large compared to the solution energies themselves, Fig. 1, as well as to the canonical structural-energy differences and first-principles results for random $4 d$ alloys, Fig. 3(b). Nonetheless, the VBA- $v^{(2)}$ model defined above provides a good fit to these first-principles results. To see this we note that (5) may be used to extract $\Delta E_{C}^{\alpha-\beta}\left(N_{d}^{C}\right)$ from the first-principles results $\Delta E_{\mathrm{sol}}^{\mathrm{bcc}-\mathrm{hcp}}$ in Fig. 2 knowing the canonical curve for the elements $\Delta E_{C}^{\text {bcc-hcp }}\left(N_{d}^{A}\right)$. Further, if the VBA- $v^{(2)}$ model is correct the energies extracted in this manner should, when plotted as functions of $N_{d}^{C}$ fall on the canonical curve. As seen in Fig. 3(a) the reproduction of the canonical curve is near perfect showing the validity 


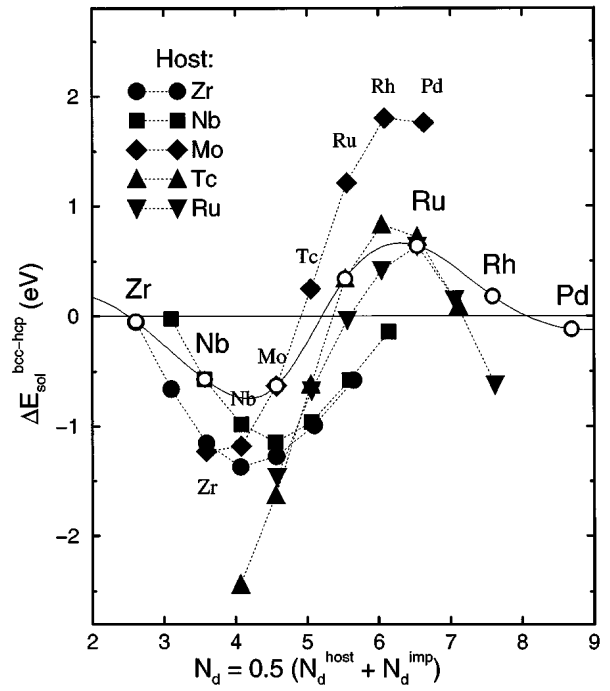

FIG. 2. The calculated bcc-hcp structural difference in the solution energy for four $4 d$ hosts, filled symbols, plotted as a function of the occupation of an impurity-host bond. A dotted line connects results for a particular host and along the line the sequence of impurities is $\mathrm{Zr}, \mathrm{Nb}, \mathrm{Mo}, \mathrm{Tc}$, and $\mathrm{Ru}$. The hosts are labeled by large letters and the impurities by smaller letters. For comparison the figure also includes the structural energies for elemental metals [11-13] here calculated in the atomic sphere approximation and indicated by a thin line.

of the VBA. The few exceptions, late $4 d$ metals in $\mathrm{Zr}$ and $\mathrm{Nb}$ hosts and early $4 d$ metals in a Mo host, are expected from the fact that pair interactions alone cannot reproduce the structural energy difference for these elements [27,28]. In fact, the introduction of higher many-body potentials into (4) improve the results of the VBA- $v^{(2)}$ model.

The fact that it is the simple average occupation $N_{d}^{C}$ rather than the concentration weighted average which enters the first term in (5) shows that, apart from the host contribution, the structural part of the impurity solution energy is governed by local effects in the form of the $d$ occupation of the impurity bonds. This local bond picture has important and unexpected consequences for the structural energy differences in random alloys which to our knowledge have not been considered in the literature. In the VCA for a random alloy all lattice sites are equivalent, and it is therefore generally assumed that the structural energy differences in such alloys may be given by the canonical curve for a pure metal at the concentration averaged $d$ occupation. The first-principles calculations included in Fig. 3(b) show that this assumption is, in fact, not correct. Instead, the structural energies are substantially reduced as a result of the random local environment. We note that the VBA$v^{(2)}$ model captures this reduction.

To explain this result as well as the local bond model for impurity solution energies we show in Fig. 4 the density of states (DOS) for the valence $d$ electrons $(d$-DOS) in MoPd for three states of different order, $B 2, B 11$, and completely random, together with the $d$-DOS for bcc Ru which is the average element corresponding to MoPd. All calculations are performed at the same volume and for

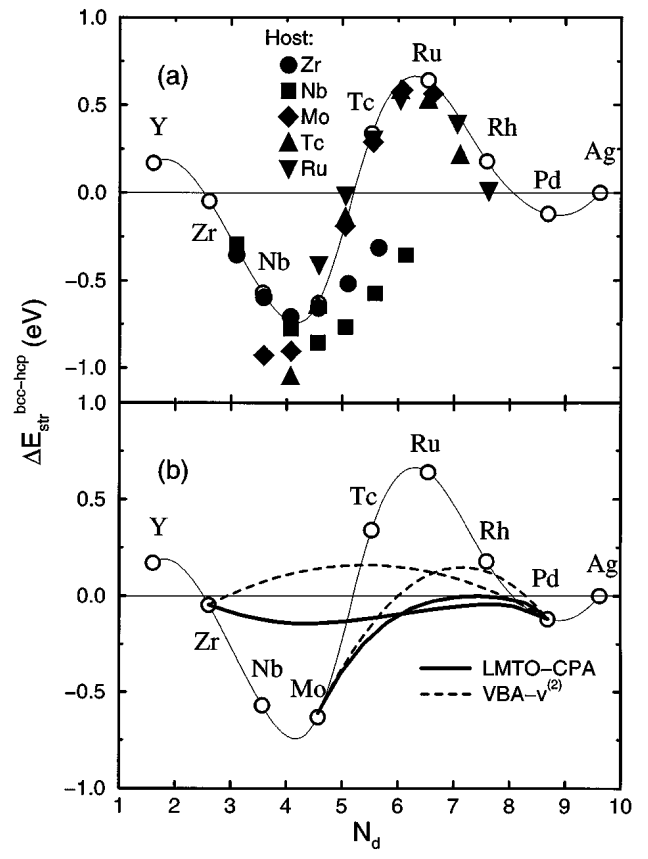

FIG. 3. (a) Comparison between the structural energy difference curve for elemental metals, thin line, and $\Delta E_{C}^{\alpha-\beta}\left(N_{d}^{C}\right)$ extracted from the first-principles results by (1), filled symbols. (b) The calculated bcc-hcp structural energy difference in random $\mathrm{Zr}-\mathrm{Pd}$ and Mo-Pd alloys, heavy lines. The results of the VBA- $v^{(2)}$ model, $\Delta E_{A_{1-c} B_{c}}^{\alpha-\beta}=2 c(1-c) \Delta E^{\alpha-\beta}\left(N_{C}^{d}\right)+(1-$ $c)^{2} \Delta E^{\alpha-\beta}\left(N_{A}^{d}\right)+c^{2} \Delta E^{\alpha-\beta}\left(N_{B}^{d}\right)$, indicated by broken lines.

the same underlying crystal lattice. In the $B 2$ structure all nearest neighbors of Mo are $\mathrm{Pd}$ atoms, i.e., all nearest neighbor bonds are Mo-Pd bonds. According to the VBA these bonds should correspond to those of $\mathrm{Ru}$, and for this reason the $d$-DOS of Ru and B2-MoPd [29] shown in Fig. 4 are very similar.

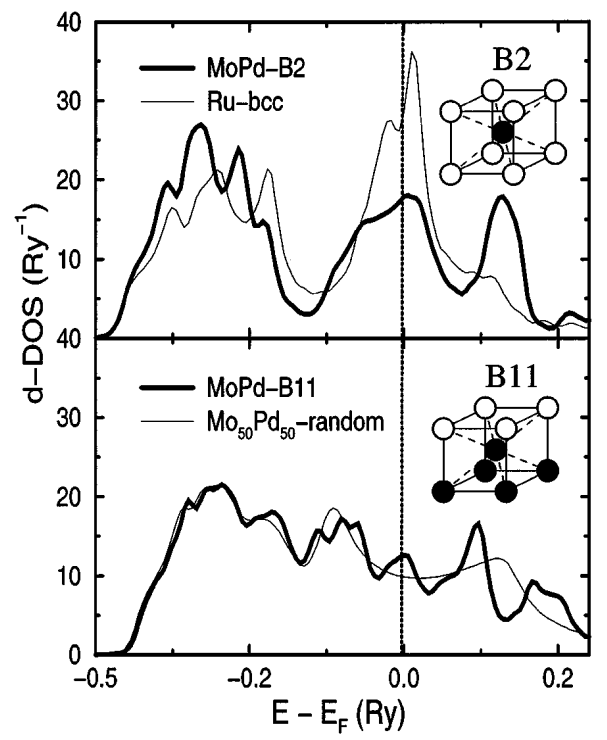

FIG. 4. Calculated $d$ state densities. The upper panel shows results for $B 2 \mathrm{MoPd}$ and the corresponding average element bcc Ru. The lower panel shows results for B11 MoPd and a completely random bcc MoPd alloy. 
On the other hand, in the $B 11$ structure every Mo atom has four Mo and four Pd as nearest neighbors and similar for Pd. Hence, the nearest neighbor environment for each atom in a two-atom nearest neighbor bond model corresponds to the completely random alloy. It is therefore not surprising that the $d$-DOS for the $B 11$ structure is very close to that of the random alloy. However, the important and unexpected point is that the $d$-DOS for the completely random MoPd alloy does not show any resemblance to that of the bcc transition metals. That is, the existence of three different types of bonds Mo-Mo, Pd-Pd, and MoPd completely destroys the electronic states responsible for stabilizing the hcp structure in this case.

In conclusion, we discuss the results of the model of enthalpy of solution based on the VCA and presented in Ref. [2], in relation to the present calculations. First, we find the solution energy of $\mathrm{Pd}$ in hep $\mathrm{Zr}$ to be $0.14 \mathrm{eV}$, cf. Fig. 1, and in bcc $\mathrm{Zr}$ to be $-0.44 \mathrm{eV}$. This is in qualitative agreement with the values in Table II-4 of Ref. [2], and leads to the prediction of a large solubility of $\mathrm{Pd}$ in bcc $\mathrm{Zr}$ and a small solubility in hep $\mathrm{Zr}$ which agrees with the experimental phase diagram. Second, we note that in the VCA the structural difference in the solution energy will be a linear function of the $d$ occupation. According to Fig. 2 this is only a good approximation in a limited $d$ occupation range. Finally, the VCA does not account for the substantial reduction of the structural energy difference caused by randomness, even in the dilute limit. Since the VCA is often assumed to hold for random alloys this result should have important consequences within alloy theory.

Center for Atomic-scale Materials Physics is sponsored by the Danish National Research Foundation. The present work was financed in part by The Danish Research Councils through The Center for Surface Reactivity.

[1] A. R. Miedema, P.F. Chatel, and F. R. de Boer, Physica (Amsterdam) 100B, 1 (1980).

[2] F.R. de Boer, R. Boom, W.C.M. Mattens, A. R. Miedema, and A. K. Niessen, Cohesion in Metals: Transition Metal Alloys (North-Holland, Amsterdam, 1988).

[3] R. Hultgren, P.D. Desai, D. T. Hawkins, M. Gleiser, and K. K. Kelley, Selected Values of Thermodynamic Properties of Binary Alloys (American Society for Metals, Ohio, 1973).

[4] B. Drittler, M. Weinert, R. Zeller, and P.H. Dederichs, Phys. Rev. B 39, 930 (1989).

[5] D. D. Johnson, D. M. Nicholson, F. J. Pinski, B. L. Gyorffy, and G. M. Stocks, Phys. Rev. B 41, 9701 (1990).

[6] A. V. Ruban and H.L. Skriver, Phys. Rev. B 55, 8801 (1997).

[7] N. Papanikolaou, R. Zeller, P.H. Dederichs, and N. Stefanou (to be published).

[8] A.R. Miedema and A. K. Niessen, CALPHAD 7, 27 (1983).

[9] A. K. Niessen and A. R. Miedema, Ber. Bunsen-Ges. Phys. Chem. 87, 717 (1983).

[10] The shape of the structural energy difference curve for the transition metals is the same for the $3 d, 4 d$, and $5 d$ series and may be derived within canonical band theory [O. K. Andersen and O. Jepsen, Physica (Amsterdam) 91B, 317 (1977)].

[11] D. G. Pettifor, in Metallurgical Chemistry, edited by O. Kubashevski (HMSO, London, 1972).

[12] O. K. Andersen, J. Madsen, U. K. Poulsen, O. Jepsen, and J. Kollar, Physica (Amsterdam) 86-88B, 249 (1977).

[13] H. L. Skriver, Phys. Rev. B 31, 1909 (1985).

[14] J. Perdew and A. Zunger, Phys. Rev. B 23, 5048 (1981); D. M. Ceperley and B. J. Alder, Phys. Rev. Lett. 45, 566 (1980).

[15] F. Ducastelle, Order and Phase Stability in Alloys (NorthHolland, Amsterdam, 1991).

[16] A. Zunger, in Statics and Dynamics of Alloy Phase Transformations, edited by P.E. A. Turchi and A. Gonis (Plenum Press, New York, 1994), p. 361.

[17] D. de Fontaine, in Progress of Theoretical Physics, edited by T. Morita, M. Suzuki, K. Wada, and M. Kaburagi (Yukawa Institute of Theoretical Physics and The Physical Society of Japan, Tokyo, 1994), p. 115.

[18] We employ Andersen's linear muffin-tin orbitals method in the atomic sphere approximation [O. K. Andersen and O. Jepsen, Phys. Rev. Lett. 53, 2571 (1984); O. K. Andersen, O. Jepsen, and D. Glötzel, in Highlights of Condensed-Matter Theory, edited by F. Bassani, F. Fumi, and M. P. Tosi (North-Holland, New York, 1985)] in conjunction with the coherent potential approximation. Both valence and core electrons were treated self-consistently in the scalar relativistic and local density approximations [14]. The screened-impurity model [A. V. Ruban, I. A. Abrikosov, and H.L. Skriver, Phys. Rev. B 51, 12958 (1995)] with a prefactor of $\beta=0.6$ was used to correct the electrostatic one-electron potential and the total energy of the LDA calculation for charge transfer effects in the single-site approximation. The atomic sphere radii of the alloy components were taken to be equal.

[19] A. I. Abrikosov and H. L. Skriver, Phys. Rev. B 47, 16532 (1993).

[20] They will be discussed in the separate paper; see also A. Christensen et al., Phys. Rev. (to be published).

[21] D. G. Pettifor, Solid State Commun. 28, 621 (1978).

[22] D. G. Pettifor, Phys. Rev. Lett. 42, 846 (1979).

[23] R. E. Watson and L. H. Bennett, Phys. Rev. Lett. 43, 1130 (1979).

[24] J. A. Moriarty, Phys. Rev. B 38, 3199 (1988).

[25] The main difference between the model used in Ref. [26] and the VBA is that in the VBA we neglect the difference in the $d$-band width of the alloy species. This may be an oversimplification in the case of alloys with components from different transition metal series, in particular, for $3 d-5 d$ alloys.

[26] A. V. Ruban and H.L. Skriver, Phys. Rev. B 55, 856 (1997).

[27] J. A. Moriarty, Phys. Rev. B 42, 1609 (1990).

[28] M. Aoki, Phys. Rev. Lett. 71, 3842 (1993).

[29] Similar unpublished results by A.R. Williams, C.D. Gellat, and V.L. Moruzzi for the $d$-DOS of B2-MoTc, $\mathrm{NbRu}$, and $\mathrm{ZrRh}$, has been used by Pettifor to justify the common $d$-band model [see D.G. Pettifor, Solid State Phys. 40, 43 (1987)]. We believe that Pettifor's model is correct if applied to the individual bonds formed by $d$ states. 